\title{
ARTÍCULO ORIGINAL \\ Características de los pacientes adultos con neutropenia febril en un hospital universitario (Medellín, 2012-2016)
}

Fecha de recibido: 19 de diciembre de 2018

Fecha de aprobación: 13 de mayo de 2019

Forma de citar este artículo: Arroyave-Peña T, PuertaRojas JD, Beltrán-Benavidez RE, Salgado-Gómez TM, Ramírez-Cadavid DC Arroyave-Ávila D, RuizCeballos AC, LondoñoAguilar LF, Jaimes-Barragán FA. Características de los pacientes adultos con neutropenia febril en un hospital universitario (Medellín, 2012-2016). Med UPB. 2019;38(2):108-113. DOI:10.18566/medupb.v38n2.a02

1 Departamento de Urgencias Hospital Pablo Tobón Uribe. Medellín, Colombia.

2 Pontificia Universidad Javeriana. Cali, Colombia.

3 Fundación Universitaria San Martín. Medellín, Colombia.

4 Grupo Académico de Epidemiología Clínica (GRAEPIC), Departamento de Medicina Interna, Facultad de Medicina, Universidad de Antioquia. Medellín, Colombia.

5 Unidad de Investigaciones, Hospital Pablo Tobón Uribe. Medellín, Colombia.

Dirección de correspondencia: Tatiana Arroyave Peña. Correo electrónico: tati2110@ hotmail.com
Characteristics of adult patients with febrile neutropenia in a university hospital (Medellin, 2012-2016) / Características dos pacientes adultos com neutropenia febril em um hospital universitário (Medellín, 2012-2016)

Tatiana Arroyave-Peña ${ }^{1,2}$, José David Puerta-Rojas ${ }^{3}$, Rafael Eduardo Beltrán-Benavidez ${ }^{2}$, Tania Margarita Salgado-Gómez ${ }^{3}$, Diana Carolina Ramírez-Cadavid³, David ArroyaveÁvila ${ }^{3}$, Ana Camila Ruiz-Ceballos ${ }^{3}$, Luisa Fernanda Londoño-Aguilar ${ }^{3}$, Fabián Alberto Jaimes-Barragán ${ }^{4,5}$

\section{RESUMEN}

Objetivo: la neutropenia febril es una urgencia médica que se presenta frecuentemente en pacientes con inmunosupresión asociada a tratamientos oncológicos, que aumenta el riesgo de complicaciones infecciosas y de morir. El objetivo de esta investigación es describir las características clínicas, microbiológicas y pronósticas de los pacientes con neutropenia febril en un hospital universitario.

Metodología: estudio observacional descriptivo, entre enero de 2012 y junio de 2016, en pacientes con diagnóstico de neutropenia febril (neutrófilos menores de $500 \mathrm{cel} /$ $\mathrm{mm}$, temperatura mayor de $38^{\circ} \mathrm{C}$ ) hospitalizados en el Hospital Pablo Tobón Uribe de Medellín, Colombia.

Resultados: se evaluaron 226 pacientes con neutropenia febril, de los que el 96\% tenía enfermedad oncológica de base. Las principales fuentes de infección fueron la piel y el tracto gastrointestinal. Del total de los hemocultivos, el 31\% fue positivo. El principal aislamiento fue Escherichia coli (E. coli) y la mortalidad hospitalaria fue del $17 \%$.

Conclusiones: las características clínicas de la población fueron similares a las de la literatura internacional. Se encontraron algunas discrepancias en cuanto a epidemiología de los microorganismos aislados, resistencia microbiana y mortalidad. Solo un tercio de los pacientes presentó bacteriemia, E. coli sigue siendo el principal patógeno.

Palabras clave: neutropenia febril; oncología médica; bacterias; antibacterianos

\section{ABSTRACT}

Objective: Febrile neutropenia is a condition present in patients with some degree of immunosuppression associated to oncology treatment, which leads to risks of infectious complications and death. The aim of this research is to describe the clinical, microbiological and prognostic characteristics in patients with febrile neutropenia in a university hospital.

Methodology: Retrospective cohort study developed between January of 2012 and June of 2016 in patients with febrile neutropenia (neutrophils $<500$ cell/mm, temperature $>38.3$ $\left.{ }^{\circ} \mathrm{C}\right)$, who were hospitalized in the Pablo Tobon Uribe Hospital of Medellin, Colombia. Results: 226 patients were evaluated, $96 \%$ of who had an oncological diagnostic. The main infectious sources were the skin and the gastrointestinal tract. Blood cultures were positive in $31 \%$. The main pathogen was Escherichia coli in $26 \%$ and the overall hospital mortality rate was $17 \%$. 
Conclusions: The clinical characteristics of our population were similar to the national and international reports, but we found epidemiological differences in microbiological agents, bacterial resistance and mortality. Only one third of the patients presented bacteremia. E. coli remains the main pathogen involved.

Key words: febrile neutropenia; medical oncology; bacteria; anti-bacterial agents

\section{RESUMO}

Objetivo: a neutropenia febril é uma urgência médica que se apresenta frequentemente em pacientes com imunossupressão associada a tratamentos oncológicos, que aumenta o risco de complicações infecciosas e de morte. O objetivo desta investigação é descrever as características clínicas, microbiológicas e prognósticas dos pacientes com neutropenia febril em um hospital universitário.

Metodologia: estudo observacional descritivo, entre janeiro de 2012 e junho de 2016, em pacientes com diagnóstico de neutropenia febril (neutrófilos menores de 500 cel/ $\mathrm{mm}$, temperatura maior de $38{ }^{\circ} \mathrm{C}$ ) hospitalizados no Hospital Pablo Tobón Uribe de Medellín, Colômbia.

Resultados: se avaliaram 226 pacientes com neutropenia febril, dos quais, 96\% tinha doença oncológica de base. As principais fontes de infecção foram a pele e o trato gastrointestinal. Do total das hemoculturas, $31 \%$ foi positivo. O principal isolamento foi Escherichia coli (E. coli) e a mortalidade hospitalar foi de $17 \%$.

Conclusões: as características clínicas da população foram similares às da literatura internacional. Se encontraram algumas discrepâncias em quanto a epidemiologia dos microrganismos isolados, resistência microbiana e mortalidade. Só um terço dos pacientes apresentou bacteriemia, E. coli segue sendo o principal patógeno.

Palavras chave: neutropenia febril; oncologia médica; bactérias; antibacterianos

\section{INTRODUCCIÓN}

La neutropenia febril es la condición en la que un paciente con inmunosupresión -con conteos bajos de leucocitos- presenta fiebre como signo clínico de un proceso infeccioso. Tiene mayor relevancia en hematología y, especialmente, en pacientes con tratamiento citotóxico, como la quimioterapia $^{1,2}$. Se considera una urgencia oncológica con una mortalidad global que oscila entre 4\% y $10 \%$, dependiendo de la enfermedad de base, el estadio inmunológico, las comorbilidades y el tipo de intervención temprana. En Colombia, según el Instituto Nacional de Salud, entre el $60 \%$ y $85 \%$ de los pacientes oncológicos cursan con esta entidad ${ }^{3}$.

La neutropenia febril (NF) se define como un conteo absoluto de neutrófilos menor de 500 células por milímetro cúbi$\mathrm{co}^{4,5}$ y una temperatura igual o mayor de $38.3{ }^{\circ} \mathrm{C}$ de forma aislada, o mayor de 38 ${ }^{\circ} \mathrm{C}$ de forma sostenida, durante una hora ${ }^{6,7}$. Se debe sospechar siempre en presencia de neutropenia febril, un posible foco infeccioso bacteriano y, ante esta premisa, considerar el inicio empírico y temprano del tratamiento antibiótico, según la clínica del paciente y la epidemiología de la región donde se encuentre.

Fuera de la relevancia clínica, la neutropenia febril implica costos para el sistema de salud, por el tiempo de estancia hospitalaria y la necesidad de unidad de cuidados intensivos derivados de ella, además de la afectación en la calidad de vida. Por lo anterior, tiene relevancia caracterizar la población que presenta este síndrome y describir su epidemiologia en Colombia, dado que la identificación y el tratamiento oportuno puede mejorar sustancialmente el pronóstico. Nuestro objetivo es describir las características básicas de una población de pacientes con diagnóstico de neutropenia febril, atendidos en un hospital universitario.

\section{METODOLOGÍA}

Estudio observacional retrospectivo en una cohorte del Hospital Pablo Tobón 
Uribe (HPTU, Medellín, Colombia), durante el periodo entre enero de 2012 y junio de 2016. La recolección de la información se realizó entre julio y diciembre de 2016. La base de datos incluía, inicialmente, 350 pacientes (historias clínicas), de los cuales se excluyeron 124. La información recopilada cumple con los siguientes criterios de inclusión: edad mayor de 18 años, conteo de neutrófilos menor de $500 \mathrm{cel} / \mathrm{mm}^{3}$ en, al menos, dos recuentos y temperatura mayor o igual a $38,3{ }^{\circ} \mathrm{C}$ o de $38{ }^{\circ} \mathrm{C}$ de forma sostenida por una hora. Se excluyeron los pacientes que tenían historia incompleta o eran remitidos de otra institución. La información se obtuvo de las historias de los hospitalizados por urgencias, con base en los códigos de la clasificación internacional de enfermedades (D70X) y los números suministrados por el departamento de gestión de la información de la institución, bajo el consentimiento institucional, para la revisión y análisis de las historias clínicas.

Se mantuvo la confidencialidad de la información de los pacientes, identificándolos con número de historia y sin datos personales. El estudio fue avalado por el Comité de Ética e Investigación de la institución y se siguieron los lineamientos de la resolución 8430 de 1993 del Ministerio de Salud colombiano ${ }^{8}$.

Para las variables cualitativas se usaron medidas de frecuencias absoluta y relativa y para las cuantitativas se utilizaron medianas, con rango intercuartílico. La organización del artículo se hizo según los parámetros de STROBE, recomendados para el reporte de estudios observacionales $^{9}$.

\section{RESULTADOS}

Se identificaron 226 pacientes que cumplían criterios de inclusión. La mediana de edad fue 49 años (rango intercuartílico =33-63), la mayoría de Antioquia (76\%),
18\% del departamento de Córdoba y los restantes del resto del país. $53.6 \%$ eran hombres, $\mathrm{n}=121$. Los que tenían diagnóstico oncológico fueron el 96.4\% ( $\mathrm{n}=218)$, de los cuales, $75 \%(n=164)$ eran de origen hematológico y el $25 \%$ restante presentaba diversos tumores sólidos; en el $90 \%$ se documentó historia reciente de quimioterapia $(\mathrm{n}=196)$.

Las comorbilidades más frecuentes fueron: hipertensión arterial $(22 \%, \mathrm{n}=50)$, infección por VIH $(6.6 \%, \mathrm{n}$ $=15)$, enfermedad hepática $(6.15 \%, \mathrm{n}=14)$, trasplante de órgano sólido $(5.7 \%, \mathrm{n}=13)$, enfermedad pulmonar obstructiva crónica $(5.3 \%, \mathrm{n}=12)$, diabetes mellitus $(5.3 \%$, $\mathrm{n}=12)$, enfermedad reumatológica $(3.5 \%, \mathrm{n}=8)$, insuficiencia cardiaca $(2.7 \%, \mathrm{n}=6)$ y enfermedad renal crónica $(1.8 \%, \mathrm{n}=4)$. El $63.2 \%$ de los pacientes $(\mathrm{n}=143)$ tenía neutropenia menor de 100 células. Otros parámetros clínicos y de laboratorio en el momento de la detección del episodio de neutropenia febril se muestran en la Tabla 1.

Se documentó como principal foco infeccioso la piel $\mathrm{y}$ los tejidos blandos $(22.5 \%, \mathrm{n}=51)$, seguido de tracto gastrointestinal $(22.1 \%, \mathrm{n}=50)$, tracto respiratorio $(17.3 \%, \mathrm{n}=39)$ y tracto urinario $(11.9 \%, \mathrm{n}=27)$. Los restantes corresponden a sistema nervioso central, catéteres y bacteremias $\sin$ foco $(\mathrm{n}=59,26.1 \%)$. Se realizaron 224 hemocultivos y se obtuvo aislamiento en $31.6 \%$; se tomaron 153 urocultivos, de los cuales, 14.3\% fueron positivos (Tabla 2). Se tomaron 11 muestras de líquido cefalorraquídeo, donde se aisló Criptococcus en una de ellas. Se realizaron también 70 cultivos de catéter con cuatro aislamientos positivos, que correspondían a $E n-$ terococcus gallinarum, Klebsiella pneumoniae, Staphylococcus epidermidis y Stenotrophomonas maltophilia. El total de resistencias encontradas en la sangre fueron tres (43\%) para Staphylococcus aureus meticilino resistente, seis (15\%) para bacilos Gram negativos productores de betalactamasas de espectro extendido y ocho (73\%) para Klebsiella pneumoniae productoras de carbapenemasas.

Tabla 1. Variables clínicas y de laboratorio relacionadas con el diagnóstico de neutropenia febril.

\begin{tabular}{lcc}
\hline Variable (unidades) & Mediana & Rango intercuartílico (faltantes) \\
\hline Presión arterial sistólica $(\mathrm{mm} \mathrm{Hg})$ & 110 & $100-120(3)$ \\
Frecuencia cardíaca (latidos/min) & 100 & $90-115(4)$ \\
Frecuencia respiratoria (respiraciones minuto) & 18 & $16-20(15)$ \\
Temperatura (grados Celsius) & 38.6 & $38.4-39(0)$ \\
Saturación de oxígeno (\%) & 96 & $93-97(73)$ \\
Proteína C reactiva $(\mathrm{mg} / \mathrm{dl})$ & 11.2 & $5.6-18.9(11)$ \\
Hemoglobina $(\mathrm{g} / \mathrm{dl})$ & 8.9 & $7.9-10.5(2)$ \\
Plaquetas (células/mL) & 38000 & 15000 a $96000(3)$ \\
\hline
\end{tabular}


Tabla 2. Microrganismos aislados según sitio de cultivo.

\begin{tabular}{lcccc}
\hline Microorganismo & \multicolumn{2}{c}{ Hemocultivo } & \multicolumn{2}{c}{ Urocultivo } \\
\hline Escherichia coli & $\mathbf{n}$ & \% & $\mathrm{n}$ & $\%$ \\
Klebsiella pneumoniae & 19 & 26.8 & 17 & 77.3 \\
Pseudomonas spp. & 11 & 15.5 & 0 & 0.0 \\
Pseudomona auruginosa & 9 & 12.7 & 0 & 0.0 \\
Staphylococcus aureus & 0 & 0 & 2 & 9.1 \\
Staphylococcus epidermidis & 7 & 9.9 & 1 & 4.5 \\
Staphylococcus haemolitycus & 2 & 2.8 & 0 & 0.0 \\
Streptococcus salivaris & 0 & 0 & 1 & 4.5 \\
Enterobacter cloacae & 2 & 2.8 & 0 & 0.0 \\
BGN sin clasificación & 0 & 0 & 1 & 4.5 \\
Otros & 6 & 8.5 & 0 & 0.0 \\
Total microorganismos & 15 & 21.1 & 0 & 0.0 \\
\hline
\end{tabular}

a Otros: Aspergillus spp., Candida krusei, Enterobacter cloacae complex, Haemophylus influenza, Leclercia adecarboxylata, Listeria monocitogenes, Micrococcus spp., Ralstonia Manitolytica, Rautella planticola, Serratia marcences, Staphylococcus hominis, Staphylococcus capitis, Streptococcus gallolyticus, Streptococo spp., Tricosporum asahit.

La mediana de tiempo de estancia hospitalaria fue de 24.8 días (rango intercuartílico $=9-30)$ y el $22.5 \%(n=51)$ requirió tratamiento de soporte en cuidados intensivos y falleció el 17\% ( $\mathrm{n}=39)$.

\section{DISCUSIÓN}

El presente estudio caracteriza la población con neutropenia febril en el Hospital Universitario Pablo Tobón Uribe de la ciudad de Medellín. Los pacientes oncológicos se presentan con neutropenia febril por el estado de inmunosupresión asociado a la quimioterapia, en especial, aquellos cuyo origen del cáncer es hematológico, con una consecuente susceptibilidad a procesos infecciosos ${ }^{10}$.

El estado clínico inicial de los participantes era aceptable, se presentan con estabilidad hemodinámica, con una mediana de presión arterial sistólica de $110 \mathrm{mmHg}$, pero taquicárdicos (frecuencia cardiaca promedio de 100 latidos por minuto) y febriles, con un promedio de temperatura de $38.7^{\circ} \mathrm{C}$.

En muchos pacientes con neutropenia febril no es posible encontrar un foco infeccioso, pues este solo es detectable en un $20 \%$ a $30 \% \%^{7,11}$, y de los hemocultivos tomados, según estudios latinoamericanos, hasta un 38\% resultan con algún aislamiento ${ }^{12}$. En este trabajo, al 99\% de los pacientes se les realizó hemocultivo, de los cuales, el $31 \%$ fueron positivos, no alejándose de la literatura actual ${ }^{12,13}$. Los focos infecciosos más comunes fueron piel y tracto gastrointestinal, otros estudios soportan estos hallazgos, incluyendo además el tracto respiratorio ${ }^{7,13}$.

Madrid C. (2013) reporta en Colombia los microrganismos Gram negativos como los más comunes y señala a $E$. coli como el patógeno más frecuente, seguido por $K$. pneumoniae y $S$. aureus ${ }^{14}$. En las referencias mundiales se evidencia el mismo comportamiento con una mayor prevalencia de infecciones por Gram negativos ${ }^{12,15,16}$, pero llama la atención el aumento en las infecciones por Gram positivos que, en algunos estudios, ocupan el primer lugar, asociadas con factores de riesgo específicos como mucositis grave, uso de catéteres vasculares por largo tiempo y uso de antibióticos profilácticos contra Gram negativos ${ }^{15-17}$. Estos factores, aparentemente, no están presentes con igual frecuencia en la población descrita y esto podría explicar la proporción de Gram positivos inferior al 20\% del total de bacteriemias.

El desarrollo de resistencia microbiana con el uso indiscriminado de antibióticos ${ }^{18}$ ha obligado a estudiar a los pacientes complejos con algún grado de inmunosupresión, en especial, se ha encontrado un aumento de la resistencia antibiótica a Gram negativos en personas con neutropenia febril ${ }^{19-21}$.

En la presente población, el 15\% de los bacilos Gram negativos eran BLEE positivo y $43 \%$ de los Staphylococcus eran meticilino-resistentes. La resistencia a antibióticos varía según la epidemiología local. Por ejemplo, Catta- 
neo C. (2008), en un estudio italiano con este tipo de pacientes, muestra una resistencia cercana al $60 \%$ y los bacilos Gram negativos resistentes son alrededor del $50 \%{ }^{17}$. En cambio, Lee J. (2016) describe para Corea que la frecuencia de resistencia a la meticilina fue mayor (88\%), mientras los BLEE positivos apenas estuvieron presentes en un $30 \%$ de los microrganismos ${ }^{18}$.

Se hace importante destacar, entonces, en nuestro estudio, comparado con la mayoría de referencias internacionales, que las resistencias a antimicrobianos aparentemente tienen una menor ocurrencia, aunque en Colombia van en ascenso desde 2010, según el Instituto Nacional de Cancerología 22 ; esto puede ser dado por la calidad y cantidad de reportes sobre resistencias y tamaños de muestra de estudios internacionales, contra los otros nacionales y el actual.

Kuderer N. (2006) encuentra una media de hospitalización de 11.5 días para sujetos con neutropenia febril en EE. UU. ${ }^{23}$, valor inferior a lo presentado en nuestro medio, 24.8 días. La mortalidad para pacientes hospitalizados con neutropenia febril en Europa se estima en $10 \%{ }^{19}$ y para Norte América, la mortalidad está entre el $4 \%$ y $10 \%{ }^{24}$; cifras que también se encuentran por debajo de lo evidenciado en el presente estudio, probablemente, debido a las mismas diferencias poblacionales, las características de los pacientes, tipo de malignidades, las comorbilidades y las complicaciones infecciosas ${ }^{3}$.

Las limitaciones del presente estudio fueron principalmente en el proceso de selección y recolección de la información, debido a pérdida de datos e historias clínicas incompletas, además, por los sistemas electrónicos de historias clínicas, que requieren de una búsqueda extensa de datos y cabe la posibilidad de omisión, proceso que puede afectar el análisis de resultados.
Para concluir, el presente reporte describe las características clínicas, microbiológicas y el pronóstico de los pacientes de una institución con neutropenia febril, encontrando similitudes y discrepancias con la literatura mundial. En el comportamiento microbiológico, en términos de aislamientos y patrones de resistencia a antibióticos, se encuentran diferencias significativas con respecto a otros reportes del país y del mundo. En este estudio se evidencia identificación de microorganismos en, aproximadamente, un tercio de la población, y los principales gérmenes aislados son Gram negativos, dentro de este grupo, la $E$. coli es la más representativa. Llama la atención el alto porcentaje de resistencia para Staphylococcus aureus y Klebsiella pneumoniae productoras de carbapenemasas, lo que constituiría una alarma sanitaria para cualquier institución, sin embargo, por el diseño metodológico del estudio, no es posible diferenciar entre asociación causal o azar, lo que amerita investigaciones especiales. Reafirmando lo expresado por la literatura, la población de mayor riesgo de presentar neutropenia febril es la oncológica, siendo el cáncer hematológico y la exposición reciente a quimioterapia, las características más repetitivas. Se debe tener especial cuidado en el enfoque de la neutropenia febril, dado que el inicio temprano de antibioticoterapia empírica, según los focos infecciosos esperados, la microbiología y las resistencias locales, puede impactar significativamente en la mortalidad de esta población.

\section{DECLARACIÓN DE CONFLICTO DE INTERESES}

Los autores declaran no tener ningún conflicto de interés.

\section{REFERENCIAS}

1. Mokart D, Saillard C, Chow-Chine BJ. Febrile neutropenia in onco-hematology patients hospitalized in Intensive Care Unit. Bull Cancer. 2015;102(4):349-359.

2. Henriquez-Camacho CV. Treatment of febrile neutropenia and prophylaxis in hematologic malignancies. Adv Hematol. 2014;2014:986938.

3. Shang J, Owolabi D. A comparison of ED and direct admission care of cancer patients with febrile neutropenia. Am J Emerg Med. 2015;33(7):966-969.

4. Godara H, Rosenstock AH. Urgencias oncológicas: Neutropenia febril. En: Manual Washington de terapéutica médica. 34. ${ }^{a}$ ed. España: Lippincott Williams and Wilkins. Wolters Kluwer Health; 2014. p. 838.

5. Health NI. Common terminology criteria for adverse events (CTCAE) [Internet]. U.S. Department of health and human services, National cancer institute; 2010. Disponible en: https://ctep.cancer. gov/protocoldevelopment/electronic_applications/docs/ctcae_v5_quick_reference_8.5x11.pdf

6. Davie A. Best practice in measurement of body temperature. Nurs stand. 2010;24:42.

7. Freifeld AG, Bow EJ, Sepkowitz KA, Boeckh MJ, Ito JI, Mullen CA, et al. Clinical Practice Guideline for the Use of Antimicrobial Agents in Neutropenic Patients with Cancer: 2010 Update by the Infectious Diseases Society of America. Clin Infect Dis. 2011;52(4):e56-93. 
8. Ministerio de Salud colombiano. resolución 8430 [Internet]. 1993. Disponible en: https://www. minsalud.gov.co/sites/rid/Lists/BibliotecaDigital/RIDE/DE/DI/RESOLUCION-8430-DE-1993.PDF

9. Vandenbroucke, J. Strengthening the Reporting of Observational Studies in Epidemiology (STROBE): Explanation and elaboration. Ann Intern Med. 2007;147(8):W163-194.

10. Mukoyama N. Retrospective survey and evaluation of first-line antibiotics for chemotherapyinduced febrile neutropenia in patients with acute myeloid leukemia. Nagoya J Med. 2017;17(79):17-18.

11. Pizzo, PA. Management of fever in patients with cancer and treatment-induced neutropenia. N Engl J Med. 1993;328:1323.

12. Villela L, González-Leal XJ. Aislamientos microbiológicos en pacientes con neutropenia febril. ¿Es apropiado el uso de las guías clínicas internacionales en México? Rev Hematol Mex. 2013;14:113-119.

13. Lakshmaiah KC. Febrile neutropenia in hematological malignancies: Clinical and microbiological profile and outcome in high risk patients. J Lab Physicians. 2015;2(7):116-120.

14. Madrid C. Epidemiología de la neutropenia febril en pacientes adultos con neoplasia hematológica, en un período de 26 meses en el Hospital Pablo Tobón Uribe, Colombia. Rev Chilena Infecto. 2013;30(2):195-201.

15. Karanwal $A B$. Review of clinical profile and bacterial spectrum and sensitivity patterns of pathogens in febrile neutropenic patients in hematological malignancies: A retrospective analysis from a single center. Indian J Med and Pediatric Oncol. 2013;2(34):85-88.

16. Lee DG, Lee JH. Increase in antibiotic-resistant Gram-negative bacterial infections in febrile neutropenic children. J Infect Chemother. 2016:3(48):181-189.

17. Cattaneo C. Recent changes in bacterial epidemiology and the emergence of fluoroquinoloneresistant Escherichia coli among patients with haematological malignancies: Results of a prospective study on 823 patients at a single institution. J Antimicrob Chemother. 2008;61(3):721728.

18. Rabagliati R. Epidemiología de neutropenia febril en pacientes adultos con leucemia aguda y linfoma. Estudio de cohorte en hospitales público y privado de Santiago, Chile. Rev Chilena Infectol. 2914;6(31):71-79.

19. Roila NJ. Management of febrile neutropenia: ESMO Clinical Practice Guidelines. Ann Onco. 2016;21(5):21-29.

20. Ramphal R. Changes in the etiology of bacteremia in febrile neutropenic patients and the susceptibilities of the currently isolated pathogens. 2004;39(1):32-40.

21. Epidemiology of bacteremia and factors associated with multi-drug-resistant gramnegative bacteremia in hematopoietic stem cell transplant recipients. Bone Marrow Transplant. 2017;39:775-781.

22. Instituto nacional de cancerología. Guía de práctica clínica para el diagnóstico y tratamiento de las infecciones bacterianas y micóticas en pacientes oncológicos mayores de 15 años con neutropenia febril posquimioterapia de alto riesgo [Internet]. 2016. Disponible en: http:// www.cancer.gov.co/images/pdf/Guias/Guias\%20de\%20Practica\%20Clinica/(GPC)-CompletaNeutropenia-Febril.pdf

23. Crawford J, Kuderer ND. Mortality, morbidity, and cost associated with febrile neutropenia in adult cancer patients. Bull cancer. 2006;106:2258-2266.

24. Rivera $D$, Brown J. The emergency care of patients with cancer: Setting the research agenda. Ann Emerg Med. 2016;2:93-101. 